\title{
INSTRUMENTOS DE AYUDA PARA MEJORAR LA VISIÓN DE PACIENTES CON ENFERMEDADES CORNEALES.
}

\section{TOOLS TO HELP IMPROVE THE VISION OF PATIENTS WITH CORNEAL DISEASES.}

\author{
Autores: ${ }^{1}$ Maritza Carolina Maldonado Vera, ${ }^{2}$ Paul Fernando Arcentales Chaw, ${ }^{3}$ Genny del \\ Carmen Arteaga Loor y ${ }^{4}$ Víctor Stiven Zevallos Cobeña. \\ 1ORCID ID: https://orcid.org/0000-0002-5513-2137 \\ ${ }^{2}$ ORCID ID: https://orcid.org/0000-0003-3338-6643 \\ ${ }^{3}$ ORCID ID: https://orcid.org/0000-0003-3307-2521 \\ ${ }^{4}$ ORCID ID: https://orcid.org/0000-0001-5155-0887 \\ ${ }^{1}$ E-mail de contacto: mmaldonado@utm.edu.ec \\ ${ }^{2}$ E-mail de contacto: pfarcentales@utm.edu.ec \\ ${ }^{3}$ E-mail de contacto: garteaga@utm.edu.ec \\ ${ }^{4}$ E-mail de contacto: vzevallos@utm.edu.ec \\ Articulo recibido:2 de Mayo del 2020 \\ Articulo revisado: 13 de Mayo del 2020 \\ Articulo aprobado: 18 de Mayo del 2020 \\ ${ }^{1}$ Licenciada en Optometría egresada de la Universidad Técnica de Manabí (Ecuador) con 10 años de experiencia laboral. \\ ${ }^{2}$ Doctor egresado de la Universidad de Guayaquil (Ecuador) con 8 años de experiencia laboral. Posee una especialización en \\ Oftalmología obtenida en la Universidad de Guayaquil (Ecuador). \\ ${ }^{3}$ Licenciada en Optometría egresada de la Universidad Técnica de Manabí (Ecuador) con 11 años de experiencia laboral. \\ Magister en Dirección y Gestión Sanitaria en la Universidad Internacional de la Rioja (España) \\ ${ }^{4}$ Licenciado en Optometría egresado de la Universidad Técnica de Manabí (Ecuador) con 10 años de experiencia laboral.
}

\section{Resumen}

Aunque la mayoría de los pacientes con discapacidad visual debido a enfermedades corneales pueden tratarse con éxito con cirugía, algunos requieren rehabilitación visual para restablecer la capacidad de lectura. Para evaluar los mejores instrumentos de ayuda para mejorar la visión, especialmente en términos de velocidad de lectura y caracterizar este grupo específico de pacientes, se realizó un ensayo cruzado aleatorio prospectivo. Los 34 pacientes se sometieron a un examen detallado (lámpara de hendidura, fondo de ojo, tomografía de coherencia óptica de dominio espectral, estudio de tratamiento precoz de la retinopatía diabética.) como prueba de detección. Solo se incluyeron pacientes con enfermedades corneales sin otras enfermedades oculares. La velocidad de lectura se evaluó con textos de velocidad de lectura consecutivamente con cinco diferentes ayudas para mejorar la visión durante un día en un diseño cruzado aleatorio. La turbidez corneal se cuantificó con densitometría corneal (Pentacam). Los pacientes tenían discapacidad visual $(\mathrm{n}=28)$, discapacidad grave $(n=4)$ o legalmente ciegos $(\mathrm{n}=2)$. Los pacientes leyeron significativamente más rápido con ayudas para mejorar la visión ( $p<0,0001)$. La velocidad de lectura más rápida se pudo lograr utilizando una lupa de video. La lupa óptica y la lupa electrónica portátil permitieron velocidades de lectura significativamente más bajas ( $\mathrm{p}<0.01$ ). En un subgrupo de pacientes (Agudeza visual $<3 / 60, \mathrm{n}=6$ ) el fondo negro permitió a los pacientes leer significativamente más rápido en comparación con el fondo blanco $(\mathrm{p}=0,03)$. Los pacientes con bajos requisitos de aumento pueden ser tratados con éxito con mejores ayudas para mejorar la visión, instrumentos ópticos y lupas electrónicas portátiles. Los pacientes más gravemente afectados necesitan de un televisor. El fondo negro permite velocidades de lectura más rápidas, probablemente debido a menos cegamiento. La discapacidad visual se puede estimar con densitometría corneal. Esta investigación confirma la capacidad de los mejores instrumentos de ayuda para mejorar la visión para restaurar con éxito la capacidad de lectura en pacientes con enfermedades corneales, que es una parte crucial de la rehabilitación visual. 
Palabras clave: Visión, Instrumentos de ayuda, Lupa, Enfermedades corneales, Neblina corneal, Densitometría corneal.

\begin{abstract}
Although most patients with visual impairment due to corneal disease can be successfully treated with surgery, some require visual rehabilitation to restore reading ability. To evaluate the best instruments to help improve vision, especially in terms of speed of reading and to characterize this specific group of patients, a prospective randomized crossover trial was performed. All 34 patients underwent a detailed examination (slit lamp, fundus, spectral domain optical coherence tomography, study of early treatment of diabetic retinopathy) as a screening test. Only patients with corneal disease without other eye diseases were included. Reading speed was evaluated with reading speed texts consecutively with five different aids to improve vision for one day in a randomized crossover design. Corneal turbidity was quantified with corneal densitometry (Pentacam). The patients had visual impairment $(n=28)$, severe disability $(n=4)$ or legally blind $(\mathrm{n}=2)$. Patients read significantly faster with aids to improve vision $(\mathrm{p}<0.0001)$. The fastest reading speed could be achieved using a video magnifier. The optical loupe and the portable electronic loupe allowed significantly lower reading speeds $(\mathrm{p}<0.01)$. In a subgroup of patients (Visual acuity $<3 / 60, n=6$ ), the black background allowed patients to read significantly faster compared to the white background $(p=0.03)$. Patients with low magnification requirements can be successfully treated with better vision aids, optical instruments, and portable electronic loupes. The most severely affected patients need a television. The black background allows for faster read speeds, probably due to less blinding. Visual impairment can be estimated with corneal densitometry. This research confirms the ability of the best vision enhancement aids to successfully restore reading ability in patients with corneal disease, which is a crucial part of visual rehabilitation.
\end{abstract}

\section{Keywords: Vision, Assistive instruments, Magnifying glass, Corneal diseases, Corneal haze, Corneal densitometry.}

\section{Sumário}

Embora a maioria dos pacientes com deficiência visual devido à doença da córnea possa ser tratada com sucesso com cirurgia, alguns requerem reabilitação visual para restaurar a capacidade de leitura. Para avaliar os melhores instrumentos para ajudar a melhorar a visão, especialmente em termos de velocidade de leitura e caracterizar esse grupo específico de pacientes, foi realizado um estudo prospectivo randomizado cruzado. Todos os 34 pacientes foram submetidos a um exame detalhado (lâmpada de fenda, fundo, tomografia de coerência óptica do domínio espectral, estudo do tratamento precoce da retinopatia diabética) como teste de triagem. Apenas pacientes com doença da córnea sem outras doenças oculares foram incluídos. A velocidade de leitura foi avaliada com textos de velocidade de leitura consecutivamente, com cinco auxílios diferentes para melhorar a visão por um dia em um projeto de cruzamento randomizado. A turbidez da córnea foi quantificada com densitometria da córnea (Pentacam). Os pacientes apresentavam deficiência visual $(\mathrm{n}=28)$, incapacidade grave ( $\mathrm{n}$ $=4)$ ou legalmente cego $(n=2)$. Os pacientes leem significativamente mais rápido com recursos para melhorar a visão $(p<0,0001)$. A velocidade de leitura mais rápida pode ser alcançada usando uma lupa de vídeo. A lupa óptica e a lupa eletrônica portátil permitiram velocidades de leitura significativamente mais baixas ( $\mathrm{p}<0,01)$. Em um subgrupo de pacientes (acuidade visual $<3 / 60, \mathrm{n}=6$ ), o fundo preto permitiu que os pacientes leiam significativamente mais rápido em comparação com o fundo branco $(p=0,03)$. Pacientes com baixa necessidade de ampliação podem ser tratados com sucesso com melhores recursos visuais, instrumentos ópticos e lupas eletrônicas portáteis. Os pacientes mais afetados precisam de uma televisão. $\mathrm{O}$ fundo preto permite velocidades de leitura mais rápidas, provavelmente devido a menos cegueira. $\mathrm{O}$ comprometimento visual pode ser estimado com a densitometria da córnea. Esta pesquisa 


\section{Ciencia y Educación (ISSN 2707-3378) \\ Vol. 1 No. 5 \\ Mayo del 2020}

confirma a capacidade dos melhores recursos para melhorar a visão de restaurar com sucesso a capacidade de leitura em pacientes com doença da córnea, que é uma parte crucial da reabilitação visual.

Palavras-chave: Visão, Instrumentos de assistência, Lupa, Doenças da córnea, Névoa da córnea, Densitometria da córnea.

\section{Introducción}

La discapacidad visual es una de las discapacidades más desafiantes del mundo. Un número cada vez mayor de personas está en riesgo de discapacidad visual causado por enfermedades oculares crónicas debido a la creciente población mundial de ancianos. (Bourne, 2017). Por lo tanto, se estima que el requisito de rehabilitación visual aumentará en un futuro cercano (Claessen, 2018). En Ecuador, se estima que hay 455289 personas con discapacidad de ellos el 11,47\% de ellos poseen algún tipo de discapacidad visual. Las causas más comunes de discapacidad visual en Ecuador son la degeneración macular relacionada con la edad, el glaucoma y la retinopatía diabética (Enríquez Carlosama, 2017) Un grupo más pequeño de pacientes sufre de discapacidad visual debido a enfermedades corneales, como opacidades corneales causadas por quemaduras térmicas / químicas, distrofias corneales y enfermedad de injerto ocular versus huésped (EICH), así como queratocono.

La mayoría de los casos se pueden tratar con éxito con métodos quirúrgicos como queratoplastia perforante, queratoplastia endotelial de membrana de Descemet, queratoplastia lamelar anterior profunda, trasplante de membrana amniótica y trasplante de células madre limbares. Sin embargo, debido a factores de riesgo ocular, como la vascularización, la presión intraocular no controlada y la uveítis, algunos pacientes no pueden ser tratados con éxito con estos procedimientos (Van Essen, 2015). Además, algunos pacientes no pueden someterse a una cirugía debido a comorbilidades, como enfermedades cardíacas o rechazar la cirugía como resultado de su edad o miedo. Incluso los pacientes que pueden ser tratados con cirugía a menudo esperan meses o años para recibir un injerto, según el procedimiento.

Los pacientes con queratocono generalmente pueden tratarse con lentes de contacto, pero algunos pacientes sufren dolor mientras usan y no pueden soportar este tratamiento. Además de estos motivos, un seguro de salud insuficiente también puede evitar que los pacientes reciban una atención médica óptima. Todos estos pacientes tienen que soportar la discapacidad visual y sus consecuencias, que comprenden, además de la discapacidad de lectura, problemas en la realización de tareas de la vida diaria y las interacciones sociales.

Estos problemas conducen a una disminución de la autosuficiencia y una mayor dependencia de parientes y personas afectuosas. (Wacker, 2018) Además, los pacientes tienen más accidentes y caídas, lo que conduce a una mayor morbilidad y mortalidad (Tirrell, 2015). Por lo tanto, los pacientes con deficiencia visual deben someterse a rehabilitación visual. Al hacer un mejor uso de la visión restante, la rehabilitación tiene como objetivo mejorar la movilidad, la capacidad de lectura y, en consecuencia, la autonomía de los pacientes. Mediante mejores instrumentos de ayuda para mejorar la visión, como lupas ópticas, lupas electrónicas de video de escritorio (circuito cerrado de televisión) y sistemas portátiles de mejora de la visión electrónica, la capacidad de lectura puede mejorarse o restaurarse y también consecutivamente la calidad de vida (Costa Rufo, 2019) 


\section{Ciencia y Educación (ISSN 2707-3378) \\ Vol. 1 No. 5 \\ Mayo del 2020}

La adaptación de mejores instrumentos de ayuda para mejorar la visión adecuado para cada paciente depende de la enfermedad, el requisito de aumento, el comportamiento anterior de lectura y otros factores individuales (Coker, 2018). Además, el uso de instrumentos de ayuda para mejorar la visión depende en gran medida de la tarea para la que se utilizará. Dado que los instrumentos electrónicos de ayuda para mejorar la visión son mucho más caros en comparación con los instrumentos ópticos, la rehabilitación visual también debería incorporar esto (Falcon Poma, 2018)

Por lo tanto, el proceso de rehabilitación visual es muy complejo y requiere mucho tiempo (Díaz Guzmán, 2017). Debido a la sensibilidad al deslumbramiento y otros problemas concomitantes, como el ojo seco, la adaptación de instrumentos de ayuda para mejorar la visión es especialmente difícil en pacientes con enfermedades corneales. Aunque se sabe que la enfermedad subyacente es importante para la rehabilitación visual, no hay datos publicados sobre estos pacientes (Costa Rufo, 2019). Esto probablemente sea el resultado de la rareza de discapacidad visual irreversible debido a enfermedades corneales. La mayoría de los estudios se centran más bien en las principales causas de discapacidad visual, como la degeneración macular relacionada con la edad (Tirrell, 2015). Los mejores ajustes de contraste también solo se han evaluado para las enfermedades de la retina (Mansour, 2014)

Para evaluar los mejores instrumentos de ayuda para mejorar la visión, es necesario medir la velocidad de lectura de los pacientes para demostrar que se puede mejorar el rendimiento de lectura y cuantificar esta mejora, según una revisión realizada por Christen, M. (2017). Esto puede hacerse mediante gráficos de oraciones individuales o párrafos, como los textos de velocidad de lectura. Elegimos los textos de velocidad de lectura para medir la velocidad de lectura, porque representa la lectura de ocio (libros, periódicos) y proporciona párrafos estandarizados que coinciden con la dificultad linguística para evaluar la velocidad de lectura en mediciones repetidas (Christen, 2017). Aparte de eso, se recomienda evaluar las preferencias y características de los pacientes, Por lo tanto, en esta investigación de tipo cruzado prospectivo y aleatorizado, el objetivo fue caracterizar a los pacientes con discapacidad visual debido a enfermedades corneales y evaluar los mejores instrumentos de ayuda para mejorar la visión para este grupo en términos de rendimiento, objetivo de lectura y calificación informada por el paciente.

\section{Métodos y materiales}

\section{Población de estudio}

Los pacientes para este ensayo fueron reclutados del Hospital provincial Verdi Cevallos ubicado en la provincia de Manabí de referencia pacientes con deficiencias visuales $y$ enfermedades corneales entre julio de 2019 y enero del 2020. Se incluyeron pacientes que cumplían con los siguientes criterios de inclusión y exclusión $(n=34)$ : discapacidad visual (mejor agudeza visual correcta<6/18) causada por un enfermedad corneal (p. ej., distrofias corneales, quemadura térmica / química, enfermedad de injerto contra huésped ocular, queratocono), sin limitación visual debido a otras enfermedades oftálmicas concomitantes (p. ej., La degeneración macular relacionada con la edad, glaucoma, etc.), capacidad de conversar, leer y escribir con fluidez, mentalmente competente, sin depresión diagnosticada, sin discapacidad física que impide operar las instrumentos de ayuda para mejorar la visión. Los protocolos de reclutamiento e investigación fueron revisados y aprobados por una comisión de ética institucional, y se obtuvo el consentimiento 


\section{Ciencia y Educación (ISSN 2707-3378) \\ Vol. 1 No. 5 \\ Mayo del 2020}

informado por escrito de todos los participantes del estudio de conformidad con la Declaración de Helsinki.

\section{Examen morfológico}

Todos los pacientes fueron sometidos a un examen oftalmológico estandarizado de ambos ojos, incluyendo biomicroscopia con lámpara de hendidura y fondo de ojo. La presión intraocular se evaluó con Tonometría de aplanamiento de Goldmann y la superficie corneal se examinó con tinción con fluoresceína. Además, a todos los sujetos se les realizó una fotografía con lámpara de hendidura, medición de agudeza visual con interferencia láser y biometría corneal (Pentacam) de ambos ojos. Para asegurar que los pacientes no tuvieran enfermedades retinianas concomitantes, se les realizo una funduscopia y tomografía de coherencia óptica de dominio espectral en todos los pacientes. Para caracterizar el estado corneal, la turbidez corneal se examinó cuantitativamente con el programa de densitometría del Pentacam. Se utilizará una cámara giratoria la cual se combinará con una cámara estática para adquirir múltiples fotografías del segmento anterior del ojo. Se utilizará una configuración de 25 escaneos en el modo de liberación automática para minimizar los errores inducidos por el examinador. Un módulo de software permitirá un análisis estandarizado de densitometría corneal.

Para ello se medirá la luz corneal retro dispersada sobre un área de $12 \mathrm{~mm}$ de diámetro y un grosor corneal completo. La densitometría corneal también se deberá medir en cuatro zonas anulares centradas en el ápice de la córnea (0-2, 2-6, 6-10 y 10-12 $\mathrm{mm}$ de diámetro). La medición de densitometría también se puede proporcionar para la capa anterior (primeros 120 $\mathrm{mm}$ ), la capa central (desde los primeros $120 \mathrm{~mm}$ hasta los $60 \mathrm{~mm}$ posteriores) y la capa posterior de la córnea $(60 \mathrm{~mm})$. La densitometría se expresa en unidades de escala de grises, que van desde una dispersión de luz mínima de 0 ( $\sin$ turbidez corneal) hasta una dispersión de luz máxima de 100 (córnea totalmente opaca). La densitometría corneal se establecerá previamente para cuantificar la manifestación de varias enfermedades corneales y, por lo tanto, se elegirá para caracterizar a el grupo de pacientes. Los exámenes con lámpara de hendidura y Pentacam fueron realizados por un oftalmólogo para garantizar la homogeneidad y la reproducibilidad. Solo se incluyeron imágenes Pentacam con buena calidad, de acuerdo con el software de garantía de calidad integrado.

\section{Examen funcional}

Después de la inscripción, los pacientes se sometieron a una evaluación visual para describir múltiples aspectos de la función visual. La agudeza visual a distancia se determinó de manera estandarizada para cada ojo (ojo derecho primero) de acuerdo con el protocolo sobre el estudio de tratamiento precoz de la retinopatía diabética. Todas las mediciones se realizaron en la misma habitación en las mismas condiciones (habitación oscura). Se utilizaron gráficos de Bailey-Lovie con iluminación interna. Para determinar la agudeza visual mejor corregida monocular a una distancia de $2 \mathrm{~m}$. La puntuación letra por letra se empleó de acuerdo con el método descrito por Ferris (1982) La refracción se realizó a 2 m con la Tabla "R" de acuerdo con el protocolo sobre el estudio de tratamiento precoz de la retinopatía diabética. Brevemente, se utilizó la lente cilíndrica esférica y negativa menos positiva o negativa consistente con la mejor agudeza visual.

Además, cerca de la agudeza visual se evaluó binocularmente a $40 \mathrm{~cm}$ utilizando tablas de Bailey-Lovie y la mejor corrección para la distancia cercana. Los resultados se presentan en unidades $\log$ MAR. Según las definiciones de la Organización Mundial de la Salud, los pacientes se clasificaron en diferentes clases de 


\section{Ciencia y Educación (ISSN 2707-3378) \\ Vol. 1 No. 5 \\ Mayo del 2020}

discapacidad visual: grado 1 (agudeza visual $<6 / 18$ ), grado 2 (agudeza visual $<6 / 60$ ), grado 3 (agudeza visual <3/60) y ceguera grado 4, (agudeza visual $\leq 1$ / 60). Para el análisis estadístico, se agrupará además el Grado 1-2 como discapacidad visual moderada y 3-4 como discapacidad visual grave. El aumento apropiado se evaluó como en la rutina clínica utilizando gráficos estandarizados con oraciones en diferentes tamaños de impresión a una distancia de $25 \mathrm{~cm}$ bajo iluminación estandarizada con la mejor corrección para la distancia cercana, descrita por otros antes (Nguyen, 2009).

Brevemente, se eligió el tamaño de impresión más pequeño que aún permitía una lectura fluida como aumento requerido, lo que indica cuánto tamaño de impresión de periódico debe ampliarse. Los instrumentos de ayuda para mejorar la visión ya poseídos y utilizados regularmente por los pacientes fueron evaluados con un cuestionario, así como su sensibilidad al deslumbramiento.

\section{Instrumentos de ayuda para mejorar la visión}

Se utilizarán textos de velocidad de lectura (IReST) para evaluar la velocidad de lectura de los pacientes. Para ser comparables entre sí, los párrafos fueron diseñados originalmente por lingüistas que combinaban contenido, longitud (párrafos, en promedio 132 palabras), dificultad (lectura entre 10 y 12 años) y complejidad linguíística. Todos los párrafos se imprimen en Times New Roman 10 puntos con un espacio entre líneas y letras similar al de la impresión de un periódico. La velocidad de lectura (palabras correctas por minuto, palabras por minuto) se evaluó primero con la mejor corrección siguiendo el protocolo descrito anteriormente (Nguyen, 2009).

En resumen, los sujetos tenían que leer el texto en voz alta lo más rápido posible sin correcciones. Las palabras mal leídas se contaron y se restaron del número total de palabras del párrafo. El tiempo se midió a partir del descubrimiento del texto para tener un punto de partida exacto. Después de esta velocidad de lectura de referencia, se probaron 5 instrumentos de ayuda para mejorar la visión utilizando diferentes párrafos adaptados a los requisitos de aumento del paciente.

El orden de las pruebas se aleatorizó mediante aleatorización en bloque para minimizar los factores de confusión. Entre las pruebas de los diferentes instrumentos de ayuda para mejorar la visión, todos los pacientes tuvieron que descansar para limitar el agotamiento y aplicar lágrimas artificiales si fuera necesario. Si el paciente no estaba acostumbrado a ninguno de los dispositivos, se realizó una sesión de entrenamiento antes de la prueba de lectura, seguida de un descanso. Los pacientes recibieron capacitación sobre cómo manejar el dispositivo y tuvieron que leer, utilizándolo para familiarizar al paciente con el instrumento de ayuda para mejorar la visión. Después de completar todas las pruebas de lectura, se pidió a los pacientes que calificaran los instrumentos de ayuda para mejorar la visión de 0 a 10 (peor - mejor).

La velocidad de lectura se midió como palabras correctas por minuto y se comparó con las tasas de lectura normativas de cada texto, proporcionadas por el grupo de estudio IReST. Para comparar los diferentes instrumentos de ayuda para mejorar la visión, los resultados se presentan como porcentaje de la velocidad de lectura normal. Por lo tanto, pequeñas diferencias de velocidad de lectura normal entre textos son irrelevantes en la comparación estadística de los instrumentos de ayuda para mejorar la visión. Esto era necesario ya que IReST ofrece textos con el mismo nivel de rendimiento, pero no cinco con el mismo nivel (máx. 4). Aun así, la diferencia máxima entre los textos utilizados fue de solo nueve palabras. Para 


\section{Ciencia y Educación (ISSN 2707-3378) \\ Vol. 1 No. 5 \\ Mayo del 2020}

permitir también la comparación con otros estudios, la velocidad de lectura también se presenta como palabras correctas y palabras por minuto.

\section{Evaluación estadística}

Para los datos métricos, se calcularon los valores medios y el rango o la media y la desviación estándar (DE \pm ) y las diferencias entre los grupos se evaluaron con la prueba $t$ de Student (dos colas), si la prueba de normalidad D'AgostinoPearson mostró una distribución normal, si no con la prueba U de Mann-Whitney. La prueba exacta de Fisher se utilizó para evaluar las distribuciones grupales de variables binarias. Se realizó un análisis de varianza con medidas repetidas (ANOVA) para examinar el efecto del tipo de instrumento de ayuda para mejorar la visión en la velocidad de lectura. Para la investigación exploratoria de la influencia de la agudeza visual basal, también se analizó la velocidad de lectura para dos grupos de participantes (agudeza visual superior e inferior a 20/400). Se han realizado regresiones lineales para probar la asociación entre parámetros funcionales y morfológicos. Se ha realizado una regresión lineal múltiple para evaluar otros factores influyentes. El nivel de significación estadística se definió en dos colas como $2 \alpha$ $<0.05$. Los valores $\mathrm{P}$ se dan con ajuste $\alpha$ para pruebas múltiples (Bonferroni). Todos los cálculos se realizaron con SPSS (IBM SPSS Statistics, Chicago, IL, EE. UU., Versión 22.0.0) y GraphPad Prism (Prism 6 para Windows, Software Inc., San Diego, CA, EE. UU., Versión $6.01)$.

\section{Resultados}

\section{Población de estudio}

El reclutamiento se llevó a cabo entre el julio de 2019 y enero del 2020. Un total de 46 pacientes con enfermedades corneales fueron reclutados por los investigadores. Doce pacientes no eran elegibles debido a enfermedades retinianas concomitantes, lo que resultó en 34 sujetos (2386 años) que participaron en esta investigación clínica. En la Tabla 1 se presenta un desglose de las características demográficas de estos participantes. La mayoría de los pacientes residían con su familia o pareja $(65 \%)$, jubilados (56\%) y habían completado una formación profesional (50\%). La discapacidad visual (grado 1 de la OMS) fue más común (82\%), mientras que la discapacidad severa (grado 3 ) y la ceguera (grado 4) fueron menos frecuentes. Las características visuales se parecían a esta clasificación (ver Tabla 2). La mayoría de los pacientes sufrían de sensibilidad severa al deslumbramiento $(65 \%)$.

Tabla 1 Características demográficas de los participantes reclutados para el estudio.

\begin{tabular}{|c|c|c|}
\hline & Muestra & \\
\hline Años (edad) & 34 & $\begin{array}{l}65,7 \pm 14,2 \\
{[23,86]}\end{array}$ \\
\hline Mujeres / Hombres & $17 / 17$ & $50 \% / 50 \%$ \\
\hline $\begin{array}{l}\text { Duración de la enfermedad } \\
\text { (años) }\end{array}$ & 34 & $10[1-76]$ \\
\hline \multicolumn{3}{|c|}{$\begin{array}{l}\text { Estado de discapacidad visual } \\
\end{array}$} \\
\hline Discapacidad visual $(\leq 20 / 63)$ & 28 & $82,3 \%$ \\
\hline $\begin{array}{l}\text { Gravemente impedido }(\leq 20 / \\
400)\end{array}$ & 4 & $11.8 \%$ \\
\hline Ciego $(\leq 20 / 1000)$ & 2 & $5.9 \%$ \\
\hline \multicolumn{3}{|c|}{ Situación residencial } \\
\hline Solo & 12 & $35.3 \%$ \\
\hline Con cónyuge / pareja / familia & 22 & $64.7 \%$ \\
\hline \multicolumn{3}{|c|}{ Estado de Empleo } \\
\hline Empleado & 9 & $26.5 \%$ \\
\hline Desempleados & 6 & $17.6 \%$ \\
\hline Retirado & 19 & $55.9 \%$ \\
\hline \multicolumn{3}{|c|}{ Educación y formación profesional. } \\
\hline Escuela secundaria & 6 & $17.6 \%$ \\
\hline $\begin{array}{ll}\text { Formación } & \text { profesional } \\
\text { completada }\end{array}$ & 17 & $50 \%$ \\
\hline $\begin{array}{lll}\begin{array}{l}\text { Calificación } \\
\text { universidad }\end{array} & \text { para } & \text { la } \\
\end{array}$ & 8 & $23.5 \%$ \\
\hline Título universitario & 3 & $8.8 \%$ \\
\hline
\end{tabular}

Fuente los autores

\section{Exámenes oftalmológicos}

Los 34 pacientes incluidos en el análisis cumplieron con los criterios de inclusión y exclusión y tenían discapacidad visual debido a una enfermedad corneal. Las exploraciones de la tomografía de coherencia óptica de dominio 
espectral no se pudieron realizar en ocho pacientes debido a la turbidez corneal de alto grado.

Tabla 2 Características visuales y síntomas

\begin{tabular}{|l|l|}
\hline Agudeza visual del ojo derecho (logMAR) & $0.70[2-0.4]$ \\
\hline Agudeza visual del ojo izquierdo (logMAR) & $1.1[\mathrm{nl}-0.6]$ \\
\hline Binocular cerca de agudeza visual & $0.63 \pm 0.2$ \\
\hline Neblina corneal (Unidades en escala de grises) & $35,7 \pm 14$ \\
\hline Necesidad de ampliación & $5[1.6-30]$ \\
\hline \multicolumn{2}{|c|}{ Sensibilidad al deslumbramiento (n) } \\
\hline Bajo & $26,5 \%$ \\
\hline Moderar & $11,8 \%$ \\
\hline Grave & $61,8 \%$ \\
\hline
\end{tabular}

Fuente los Autores

Con la ayuda de fondo de ojo y medición de agudeza visual con interferencia láser se aseguró que los pacientes no tenían una enfermedad concomitante que causara discapacidad visual. Las exploraciones de Pentacam se pudieron realizar en 63 ojos. La neblina corneal era demasiado densa en 9 pacientes. En seis casos, los escaneos de Pentacam tuvieron que descartarse porque no se podían lograr los estándares de calidad. Por lo tanto, 56 ojos $(75,7 \%)$ se incluyeron en el análisis final. Las medidas de todas las capas para la zona anular central $(0-6 \mathrm{~mm})$ se consideraron las más importantes para la agudeza visual y, por lo tanto, se utilizaron para análisis adicionales. Se realizó una regresión lineal para evaluar si la turbidez corneal cuantificada se correlaciona con la agudeza visual. El modelo mostró una correlación significativa $(\mathrm{F} 1,55=11.8 ; \mathrm{p}=$ $0.001, r=-0,45)$ : un mayor grado de turbidez corneal resultó en una disminución de la agudeza visual. Para explorar la variabilidad inexplicable, se realizó una regresión lineal múltiple y se agregó secuencialmente la edad y la medición de agudeza visual con interferencia láser. Este modelo, en el que la turbidez corneal seguía siendo la variable independiente más importante, podía predecir un mayor porcentaje de la variabilidad (F 2,55 = 10,1; $\mathrm{p}<0,0001, \mathrm{r}=0,41)$, pero aún mostraba un amplio rango.
Tabla 3 Patologías oculares primarias entre los participantes (n $=34$ )

\begin{tabular}{|c|c|}
\hline \multicolumn{2}{|c|}{ Diagnóstico primario } \\
\hline Quemadura térmica / química & 77 \\
\hline Distrofia corneal & 66 \\
\hline Úlcera & 66 \\
\hline Queratocono & 3 \\
\hline $\begin{array}{l}\text { Enfermedad de injerto contra } \\
\text { huésped }\end{array}$ & 3 \\
\hline Otro & 99 \\
\hline
\end{tabular}

Fuente los Autores

\section{Ayuda para mejorar la visión}

La mayoría de los pacientes recibieron instrumentos de ayuda para mejorar la visión de manera adecuada (79\%). Sin embargo, 10 pacientes tenían o no $(\mathrm{n} A B V=3)$ o insuficientes $(\mathrm{n}=7)$. Los pacientes con discapacidad visual moderada (OMS 1-2) fueron ayudados principalmente por instrumentos ópticos de ayuda para mejorar la visión, mientras que los pacientes con discapacidad visual grave (OMS 3-4) tuvieron instrumentos electrónicos de ayuda para mejorar la visión significativamente (ver Tabla 4).

Tabla 4 Instrumentos de ayuda para mejorar la visión de los pacientes agrupados por grado de discapacidad visual.

\begin{tabular}{|l|l|l|l|}
\hline $\begin{array}{l}\text { Instrumentos de ayuda para } \\
\text { mejorar la visión }\end{array}$ & $\begin{array}{l}\text { OMS } \\
\text { Grado } \\
1-2 \\
(n=28)\end{array}$ & $\begin{array}{l}\text { OMS } \\
\text { Grado } \\
6)\end{array}$ & Valor $\mathrm{p}$ \\
\hline $\begin{array}{l}\text { Sin instrumentos de ayuda } \\
\text { para mejorar la visión }\end{array}$ & $11 \%$ & & $0.93^{\mathrm{a}}$ \\
\hline $\begin{array}{l}\text { Insuficientes instrumentos de } \\
\text { ayuda para mejorar la visión }\end{array}$ & $18 \%$ & $33.3 \%$ & $0.21^{\mathrm{a}}$ \\
\hline $\begin{array}{l}\text { Instrumentos ópticos de } \\
\text { ayuda para mejorar la visión }\end{array}$ & $89 \%$ & $83.3 \%$ & $0.55^{\mathrm{a}}$ \\
\hline Cantidad por paciente & $1[1,6]$ & $1[0,2]$ & $0.46^{\mathrm{a}}$ \\
\hline $\begin{array}{l}\text { Instrumentos electrónicos de } \\
\text { ayuda para mejorar la visión }\end{array}$ & $25 \%$ & $88,3 \%$ & $0.01^{\mathrm{a}}$ \\
\hline Cantidad por paciente & $0[0,3]$ & $1[0,3]$ & $0.04^{\mathrm{b}}$ \\
\hline Televisor & $14 \%$ & $66,7 \%$ & $0.02^{\mathrm{a}}$ \\
\hline $\begin{array}{l}\text { Sistemas } \\
\text { portátiles para mejorar la } \\
\text { visión }\end{array}$ & $14 \%$ & $50 \%$ & $0.09^{\mathrm{a}}$ \\
\hline Electrónica de consumo & $46 \%$ & $83,3 \%$ & $0.18^{\mathrm{a}}$ \\
\hline Cantidad por paciente & $0[0,4]$ & $1[0.2]$ & $0.34^{\mathrm{b}}$ \\
\hline Smartphone & $46 \%$ & $83,3 \%$ & $0.18^{\mathrm{a}}$ \\
\hline Tableta & $21 \%$ & $50 \%$ & $0.31^{\mathrm{a}}$ \\
\hline Cámara & $14 \%$ & & $0.77^{\mathrm{a}}$ \\
\hline Gafas de filtro de corte & $25 \%$ & $16,7 \%$ & $0.66^{\mathrm{a}}$ \\
\hline
\end{tabular}

Fuente los Autores

Leyenda a: prueba de Fishers b: prueba de Mann-Whitney 


\section{Ciencia y Educación (ISSN 2707-3378) \\ Vol. 1 No. 5 \\ Mayo del 2020}

Curiosamente, la mitad de los pacientes utilizaron productos electrónicos de consumo como teléfonos inteligentes y tabletas para ampliar los textos. Como razones principales, informaron que utilizan dispositivos que son menos estigmatizantes y más convenientes, ya que se llevan con ellos de todos modos (especialmente los teléfonos inteligentes).

Antes de probar la velocidad de lectura, se evaluó el requisito de aumento en todos los pacientes (Tabla 2) y los instrumentos de ayuda para mejorar la visión se adaptaron en consecuencia. La prueba de lectura se realizó con cinco instrumentos de ayuda para mejorar la visión diferente en un orden aleatorio para evaluar con cuál de ellos los pacientes logran una mejor velocidad de lectura (ver Tabla 5 para velocidades de lectura). Por adelantado, la lectura se evaluó con la mejor corrección sin ningún instrumento de ayuda para mejorar la visión. Solo seis pacientes $(16 \%)$ podían leer a intervalos de $33 \mathrm{~cm}$, mientras que todos los pacientes podían leer con instrumentos de ayuda para mejorar la visión apropiados. La lectura con un instrumento óptico de ayuda para mejorar la visión y sistemas portátiles de mejora de la visión electrónica fue imposible en siete y dos pacientes, respectivamente. Mientras que la lupa óptica podría permitir una lectura fluida promedio (80 palabras por minuto) para pacientes con un máximo de requisito de aumento de 5 veces, esto no fue posible para pacientes con $\geq 6$ veces mostraron una velocidad de lectura significativamente disminuida ( $p$ $<0,0001)$.

El mejor rendimiento de lectura para todos los pacientes se obtuvo cuando se utilizó el televisor. La velocidad de lectura fue significativamente mayor con el televisor y cualquier contraste en comparación con los instrumentos ópticos de ayuda para mejorar la visión y sistemas portátiles de mejora de la visión electrónica $(F 2,76=24.1$; $\mathrm{p}<0.001$, ANOVA). No se encontraron diferencias significativas entre los ajustes de contraste. La velocidad de lectura normal (> 90\%) solo podría lograrse en dos pacientes con un requisito de aumento de 2 veces. "Sin capacidad de lectura" se calificó como se describió anteriormente como 0 palabras por minuto para comparar la velocidad de lectura con y sin instrumentos de ayuda para mejorar la visión.

Tabla 5 Velocidad de lectura (palabras correctas por minuto, palabras por minuto) con diferentes instrumentos.

\begin{tabular}{|l|l|}
\hline \multicolumn{2}{|c|}{ Televisión } \\
\hline Mejor corrección
\end{tabular}

Con los instrumentos de ayuda para mejorar la visión, la velocidad de lectura aumentó significativamente en un 49\% ( $p<0,0001$ ). La velocidad de lectura también se analizó con pacientes asignados a dos grupos según su grado de discapacidad visual (OMS 1-2 versus OMS 3-4). El primer grupo, compuesto solo por pacientes con discapacidad grave $(n=4)$ y ciegos $(\mathrm{n}=2)$, mejoró su velocidad de lectura con instrumentos de ayuda para mejorar la visión en comparación con la mejor corrección significativamente en menor medida que el grupo menos afectado (32\% frente a 59\%, p = 0.04). Los pacientes con discapacidad visual grave mostraron diferencias significativas en el análisis de diferentes configuraciones de contraste e instrumentos de ayuda para mejorar la visión: los pacientes podían leer significativamente más rápido si el fondo era negro (sin diferencias significativas entre el color de fuente blanco o verde) en comparación con un televisor con contraste normal $(p=0.03)$ y 


\section{Ciencia y Educación (ISSN 2707-3378) \\ Vol. 1 No. 5 \\ Mayo del 2020}

nuevamente comparado con los instrumentos ópticos de ayuda para mejorar la visión y sistemas portátiles de mejora de la visión electrónica ( $\mathrm{p}<0.001)$. El segundo grupo (OMS $3-4, \mathrm{n}=28$ ) mostró los mismos resultados que el análisis de todos los pacientes.

La calificación informada por el paciente se parecía principalmente a los resultados de la evaluación de la velocidad de lectura. Sin embargo, la mayoría de los pacientes prefirió polaridad invertida a la normal $(\mathrm{p}=0.02) \mathrm{y}$ contraste verde sobre negro $(\mathrm{p}=0.006)$. Curiosamente, los pacientes calificaron los sistemas portátiles de mejora de la visión electrónica significativamente mejor que los instrumentos ópticos de ayuda para mejorar la visión $(\mathrm{p}=0,004)$.

En el grupo de pacientes con discapacidad severa, la polaridad invertida también se calificó significativamente mejor en comparación con el contraste normal $(\mathrm{p}=0.02)$ con una mayor diferencia media entre ambos. El contraste verde sobre negro se calificó ligeramente peor en comparación con la polaridad invertida, pero mejor en comparación con el contraste normal (ambos sin importancia). En pacientes con deficiencia visual moderada no hubo diferencias significativas en las calificaciones entre los tres ajustes de contraste.

Ambos instrumentos de ayuda para mejorar la visión portátil también fueron significativamente peor calificados en comparación con el uso del televisor $(\mathrm{p}=0.01)$.

Para evaluar qué parámetros funcionales influyen en la velocidad de lectura, se realizó regresiones lineales. El análisis reveló que la velocidad de lectura está relacionada con la agudeza visual cercana $(\mathrm{F} 1,34=24,7 ; \mathrm{p}=0,001$, $\mathrm{r}=0,49)$ y el requisito de aumento $(\mathrm{F} 1,34=23,1$; $\mathrm{p}<0,0001, \mathrm{r}=-0,51)$. La velocidad de lectura disminuyó con mayor aumento y menor agudeza visual cercana. La edad, por el contrario, no mostró correlación significativa $(\mathrm{F} 1,34=0.7$; $\mathrm{p}$ $=0.46, \mathrm{r}=-0.11)$. Para caracterizar aún más la cohorte de pacientes, también se evaluaron la correlación de la velocidad de lectura con la turbidez corneal. La regresión lineal mostró una correlación significativa $(\mathrm{F} 1,34=6.8 ; \mathrm{p}=0.01, \mathrm{r}$ $=-0.20)$. Una mayor opacificación de la córnea conduce a una menor velocidad de lectura.

\section{Discusión de los resultados}

En esta investigación clínica de carácter prospectivo, aleatorizado y cruzado, permitió obtener una evaluación detallada de pacientes con discapacidad visual debido a enfermedades corneales y los mejores instrumentos de ayuda para mejorar la visión para ellos en términos de velocidad de lectura y calificación informada por el paciente.

Los resultados muestran que la rehabilitación visual de estos pacientes específicos también se puede lograr con instrumentos de ayuda para mejorar la visión elegidos adecuadamente y complementando los informes anteriores de tratamiento de enfermedades de la retina. Mientras que solo seis pacientes podían leer sin un instrumento de ayuda para mejorar la visión, todos los pacientes podían hacerlo con la ayuda de un instrumento de ayuda para mejorar la visión.

Esto es similar a los hallazgos en la degeneración macular relacionada con la edad y confirma el gran valor de la rehabilitación visual a través de la provisión cuidadosa y adecuada de instrumentos de ayuda para mejorar la visión. Dado que la lectura es una parte integral de muchas actividades, la restauración de la lectura también puede beneficiar a otras partes de la vida diaria y a la independencia y calidad de vida consecutivamente. 


\section{Ciencia y Educación (ISSN 2707-3378) \\ Vol. 1 No. 5 \\ Mayo del 2020}

Proporcionar el instrumento apropiado puede ayudar a mejorar la visión.

Dependiendo del grado de discapacidad visual, se podría demostrar que diferentes instrumentos de ayuda para mejorar la visión son efectivos para aumentar la velocidad de lectura de los pacientes. Esto se ha demostrado previamente también para pacientes con enfermedades de la retina (Bourne, 2017).

Por lo tanto, la rehabilitación visual adecuada necesita el instrumento apropiado puede ayudar a mejorar la visión: los pacientes gravemente afectados (requisito de aumento de $\geq 6$ veces) no podían leer en absoluto con instrumentos portátiles, o solo mal y necesitaban una pantalla más grande (televisor) para restaurar su capacidad de lectura. Por el contrario, los pacientes con un bajo requisito de aumento podían leer con fluidez con un instrumento óptico.

Esto está en concordancia con la rehabilitación visual de la población (Christen, 2017) enfatizando nuevamente la necesidad de evaluar el requisito de aumento antes de prescribir un instrumento apropiado puede ayudar a mejorar la visión para asegurar una rehabilitación visual rentable. Entre ambos los instrumentos de mano probados no hubo diferencias significativas en la velocidad de lectura, pero los pacientes calificaron los sistemas portátiles de mejora de la visión electrónica. significativamente mejor que la lupa óptica. Esto probablemente se deba al hecho de que la lupa óptica con mayor potencia de aumento restringe el campo de visión de manera distintiva. Además de eso, los sistemas portátiles de mejora de la visión electrónica permiten el manejo en la distancia habitual y un mejor contraste (Costa Rufo, 2019).

A pesar de eso, todos los pacientes podían leer más rápido con una pantalla más grande. Este también fue el caso en un ensayo reciente de
Christen, (2017) con pacientes con degeneración macular relacionada con la edad, así como en otros ensayos previos con pacientes con enfermedad principalmente retiniana (Coker, 2018). La superioridad del televisor sobre los instrumentos portátiles de ayuda para mejorar la visión probablemente se deba al campo más amplio para la visualización, lo que es especialmente beneficioso con mayores aumentos, ya que se pueden ver más palabras simultáneamente.

En general, los televisores parecen ideales para la lectura de ocio, ya que ofrecen visualización binocular en una distancia de trabajo habitual y configuraciones de aumento y contraste variables. Sin embargo, incluso con los televisores disponibles, las expectativas de los pacientes deben manejarse con cautela, ya que solo dos pacientes podrían alcanzar aproximadamente la velocidad de lectura de una cohorte normal incluso con el televisor. Por lo tanto, se debe informar a los pacientes que los instrumentos para mejorar la visión no pueden compensar completamente una discapacidad visual para asegurar su motivación. Además, los televisores unen a los pacientes a una sola ubicación y son más caros, por lo que solo pueden verse como una adición a un instrumento de ayuda para uso móvil en tiendas, bancos, etc.

Curiosamente, ya la mitad de los pacientes utilizaron productos electrónicos de consumo como los teléfonos inteligentes como ayuda de aumento, porque son menos estigmatizantes que los instrumentos de ayuda clásicos y proporcionan las características básicas de un sistema portátil de mejora de la visión electrónica. Dado que muchos pacientes especialmente jóvenes poseen estos dispositivos de todos modos, deben ser informados sobre las posibilidades que brindan. Para concluir, el instrumento de ayuda para mejorar la visión para un paciente debe elegirse de acuerdo con su 


\section{Ciencia y Educación (ISSN 2707-3378) \\ Vol. 1 No. 5 \\ Mayo del 2020}

requisito de aumento, rendimiento de lectura y preferencia personal.

En principio, la rehabilitación visual debe apuntar a un resultado óptimo. Solo después de probar los posibles instrumentos de ayuda para mejorar la visión, un paciente puede decidir junto con el especialista, qué dispositivo es apropiado para él. Una limitación de la prueba es que no se logró evaluar el uso a largo plazo de cada dispositivo.

Los estudios futuros deberían considerar este aspecto y permitir fases en el hogar y visitas de seguimiento, ya que las pruebas en la clínica son un entorno artificial y solo el uso diario permite al paciente probar realmente un instrumento de ayuda para mejorar la visión.La hipótesis específica primera, evidenció una relación entre el clima organizacional, que a menudo cuenta con ambientes idóneos, aunque con una escasa motivación; y la preparación y desarrollo de la enseñanza, considerado por la mayoría de estar de acuerdo, a pesar de estar disconforme en la poca frecuencia en la que se usa los softwares, incluso un porcentaje menor se consideran estar en total desacuerdo con respecto a la organización del contenido y los medios para la enseñanza. Por consiguiente, la falta motivación en el trabajo docente limita el desarrollo académico, puesto que no se está innovando en cuanto a los medios de enseñanza, como la poca frecuencia en la que los softwares son utilizados en clases.

\section{Conclusiones}

La capacidad de lectura en pacientes con enfermedades corneales se puede restaurar con éxito mediante el uso de instrumentos de ayuda, lo cual depende del grado de discapacidad visual, evaluado como requisito de aumento o agudeza visual cercana. Se podrían lograr velocidades de lectura más altas con los televisores, especialmente con fondo negro, probablemente debido a un menor deslumbramiento. Los pacientes gravemente afectados calificaron la polaridad invertida como mejor, los pacientes menos afectados también podrían mejorar su velocidad de lectura lo suficiente con los instrumentos portátiles de ayuda para mejorar la visión. La cuantificación de la turbidez corneal puede ayudar a estimar la agudeza visual y el rendimiento de lectura.

\section{Referencias bibliográficas}

Bourne, R. R. (2017). Magnitude, temporal trends, and projections of the global prevalence of blindness and distance and near vision impairment: a systematic review and meta-analysis. The Lancet Global Health, 5(9), e888-e897.

Christen, M. \&. (2017). The effect of magnification and contrast on reading performance in different types of simulated low vision. Journal of Eye Movement Research JEMR, 10(2).

Claessen, H. K. (2018). Markedly decreasing incidence of blindness in people with and without diabetes in southern Germany. Diabetes care, 41(3), 478-484.

Coker, A. H. (2018). Rehabilitation referral for patients with irreversible vision impairment seen in a public safety-net eye clinic. JAMA ophthalmology, 136(4), 400-408.

Costa Rufo, C. (2019). Revisión bibliográfica sobre los resultados obtenidos con el uso de ayudas electrónicas y ópticas de baja visión en pacientes con defecto de campo central.

Díaz Guzmán, E. C. (2017). Experiencia de la Atención Primaria de Salud en el proceso de rehabilitación visual en la provincia de Santi Spíritus. Revista Cubana de Oftalmología, 30(3), 1-11.

Enríquez Carlosama, E. C. (2017). Las discapacidades en el Ecuador, situación actual. Programas y servicios de atención en salud y educación. Programas y servicios en protección legal, economía, seguridad social e inserción laboral (Master's thesis).

Falcon Poma, R. P. (2018). Condición laboral de las personas con discapacidad visual en Lima Metropolitana-2018. 
Ferris III, F. L. (1982). New visual acuity charts for clinical research. American journal of ophthalmology, 94(1), 91-96.

Mansour, B. R.-B. (2014). Validation of a lowcost wearable accelerometer for temporal gait parameter quantification. Computer methods in biomechanics and biomedical engineering, 17(S1), 160-161.

Nguyen, N. X.-K. (2009). Improvement of reading speed after providing of low vision aids in patients with age-related macular degeneration. Acta ophthalmologica, 87(8), 849-853.

Tirrell, G. S.-o. (2015). Evaluation of older adult patients with falls in the emergency department: discordance with national guidelines. Academic emergency medicine, 22(4), 461-467.

Van Essen, H. R. (2015). Matching for Human Leukocyte Antigens (HLA) in corneal transplantation-To do or not to do. Progress in retinal and eye research, 46, 84-110.

Wacker, K. B. (2018). Patient-Reported Visual Disability in Fuchs' Endothelial Corneal Dystrophy Measured by the Visual Function and Corneal Health Status Instrument. Ophthalmology, 125(12), 1854-1861.

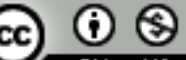
Creative Commons Reconocimiento-No Comercial 4.0 Internacional. Copyright (c) Maritza Carolina Maldonado Vera, Paul Fernando Arcentales Chaw, Genny del Carmen Arteaga Loor y Víctor Stiven Zevallos Cobeña 
